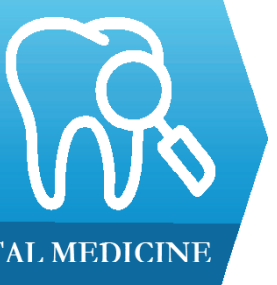

1) Department of Prosthodontics, Faculty of Dental Medicine, Iuliu Hatieganu University of Medicine and Pharmacy, Cluj-Napoca, Romania

2) Department of Periodontology, Faculty of Dental Medicine, Iuliu Hatieganu University of Medicine and Pharmacy, Cluj-Napoca, Romania

\section{Temporomandibular dysfunction diagnosis by means of computerized axiography}

\author{
Smaranda Buduru ${ }^{1}$, Silvia Balhuc ${ }^{1}$, Alexandru Ciumasu ${ }^{1}$, Andreea Kui ${ }^{1}$, \\ Cosmin Ciobanu ${ }^{2}$, Oana Almasan ${ }^{1}$, Manuela Manziuc ${ }^{1}$, Marius Negucioiu ${ }^{1}$
}

\begin{abstract}
Background and aims. The objective of this study was to evaluate the accuracy of the CADIAX Compact 2 axiograph in confirming the presumptive clinical diagnosis of temporomandibular dysfunction, established according to the muscular-articular clinical examination.

Methods. A retrospective analytical study was carried out on 50 patients, aged between 21 and 62 years, 31 women and 19 men. A clinical diagnosis was established after masticatory muscles, temporomandibular joints and mandibular border limit movements were assessed. During the same day a computerized axiography (Cadiax Compact 2, Gamma, Vienna, Austria) was performed in order to confirm the diagnosis.

Results. The study had a sensitivity of $100 \%$, which means that the CADIAX device detected all clinically positive patients. On the other hand, the specificity of the study was $74 \%$, representing the percentage of patients negatively diagnosed by CADIAX device out of the total number of patients clinically diagnosed as negative. The $88 \%$ accuracy shows the proportion in which the CADIAX device provides a diagnosis equal to the clinical one.

Conclusions. Of all the methods for investigating temporomandibular joint dysfunction, axiography is less invasive, does not irradiate the patient, it is relatively easy to use and it offers functional information about the TMJ function.
\end{abstract}

Keywords: temporomandibular disorder, disk displacement, axiography

\section{Background and aims}

Temporomandibular dysfunction is a very frequent pathology that raises important diagnostic concerns and, thus, requires careful treatment planning and selection. A precise diagnosis of the temporomandibular dysfunction type, in addition to clinical examination according to a well-established protocol, requires a series of complementary examinations: radiographs, ultrasound, cone-beam computer tomography (CBCT), MRI (which provides structural information), axiography (condylography) and assessing mandibular movements (which provide functional information) [1,2].
Diagnosis is a difficult issue because there is no consistent agreement on defining the type of temporomandibular dysfunction. None of the para-clinical examination methods is infallible, therefore they must be compared with clinical examination. MRI remains the gold standard in diagnosing musculoskeletal disorders, but this examination often remains prohibitive because of its costs, addressability, and discomfort during the process.

Examination with a computerized axiograph may be a much more convenient option for patients and eliminates the risk of radiation (CT scans, other kinds of X-ray exposures). It is more 
accessible to a dental office than other imaging devices (ultrasound, MRI) and can be a valuable tool in a more accurate diagnosis of the types of intra-articular disorder. In the literature, sensitivity and accuracy of computer axiography do not present consistent relevant data in order to be used in the diagnosis of temporomandibular dysfunctions [2-4].

The aim of this study was to evaluate the accuracy of the CADIAX Compact 2 axiograph in confirming the presumptive clinical diagnosis, established according to the muscular-articular clinical examination.

\section{Methods}

The study was performed between March 2019 and December 2019 in a private dentistry clinic in ClujNapoca. All subjects included signed an informed consent in order to use their protected medical data in clinical research.

The data collection was performed from the medical files of the patients who presented for examination and treatment.

The inclusion criteria were as follows: (1) patients who presented signs of temporo-mandibular dysfunction according to RDC/TMD, (2) no comorbidities, (3) no extensive edentulisms (which would cause instability of the axiograph's fork), (4) no dramatic limitations of mouth opening, and compliance. The exclusion criteria were patients' muscular instability and inability to correctly perform mandibular movements.

The protocol for examination was the same for each patient addressing to the clinic for diagnosis and treatment. Each subject was clinically examined by a prosthodontic specialist and, later in the same day, a computer axiography was performed by another prosthodontic specialist. The second practitioner did not receive any information about the clinical diagnosis established prior to the paraclinical examination.

\section{Clinical examination}

The presumptive clinical diagnosis was established according to the examination of the muscles, of the temporomandibular joint (TMJ) and of the mandibular borderline movements.

For the muscle examination: palpation was performed uni-digitally or bi-digitally and was carried out simultaneously (right-left) and in a comparative manner, by palpating the muscular body and/or the muscular insertions. The state of tenderness or pain, contracture or hypertension of a muscle or group of muscles was observed and noted. The muscles analyzed were the masticatory muscles (maseter, temporal, lateral and medial pterygoid, digastric, mylohyoid) in the manner described by other studies [5-7].

Temporomandibular joint (TMJ) examination technique included the classical methods of assessment, namely inspection, palpation and auscultation. The existence of pain, symmetry and amplitude of condylar movements, joint clicking and joint noises (clacking, crepitus) were examined.

Mandibular border movements (maximum opening, maximum protrusion and maximum lateral movement) were also examined. In the analysis of the movements, 2 parameters were assessed: quantity (expressed in $\mathrm{mm}$ ) and quality (rectilinear, sinusoidal, or deviated), using the same protocol as other studies mentioned [5-7].

Analyzing the clinical data, the following presumptive diagnoses were elaborated: muscles and TMJ were considered to be normal when no deviation from the functional limits was detected; uni-/bilateral disc displacement with reduction ( $D D w R$ ) was diagnosed when the amplitude of mouth opening remained unchanged, but there was a sinusoidal mandible trajectory and uni-/ bilateral articular clicking and joint jump; bilateral disc displacement without reduction (DDwoR) was diagnosed when the amplitude of the mouth opening was significantly reduced, without deviating the mandibular movement from the midline, without clicking and joint noises; unilateral disc displacement without reduction (DDwoR) was diagnosed when the mouth opening amplitude was reduced, while the mandible deviated towards the affected side; and finally, bilateral subluxation ("open lock") was the diagnosis when mouth opening amplitude was greatly increased, while movement trajectory remained rectilinear [5-7].

\section{Computer axiography analysis}

After complete clinical examination, all the subjects underwent axiography using the CADIAX Compact 2 device (Gamma, Vienna, Austria) (Figure 1). Before the examination, all patients were informed about the movements they were supposed to perform.

Computerized axiography is a system designed for analyzing the functional movements of the mandible in all three spatial dimensions. These are: the $\mathrm{X}$ axis situated sagitally, horizontally, and considered positive towards the front and negative towards the rear; the $\mathrm{Y}$ axis - situated transversely, horizontally, and considered positive outwards and negative inwards; and the $\mathrm{Z}$ axis - situated frontally, vertically, and considered positively downwards $[8,9]$.

The system's principle of operation is based on electronic recording of the movements occurring in the TMJ during mandibular movements. Recording plates provided with electromagnetic sensors are activated by the gentle contact of the writing pads, which transmit data to the central unit. The gathered information is then processed and displayed on a computer screen in the form of graphs that show the path of condylar displacement, corresponding to all 


\section{Dental Medicine}

directions of mandibular movement [10-12].

According to the recorded graphs, different diagnoses can be obtained as follows:

(1) Normal TMJ: clear, regular, superimposed graphics; a minimum quantity of $14 \mathrm{~mm}$ for opening, $9 \mathrm{~mm}$ for maximum protrusion and maximum lateral movement.

(2) Uni-/Bilaterally disc displacement with reduction (DDwR): the graphs for the incursive and excursive mandibular movements do not overlap, but the movement is not limited from a quantitative point of view (Figure 2).

(3) Uni-/Bilaterally displacement without reduction (DDwoR): significant quantitative limitation, with a straight, linear pathway, uni- or bilaterally in all mandibular movements (Figure 3).

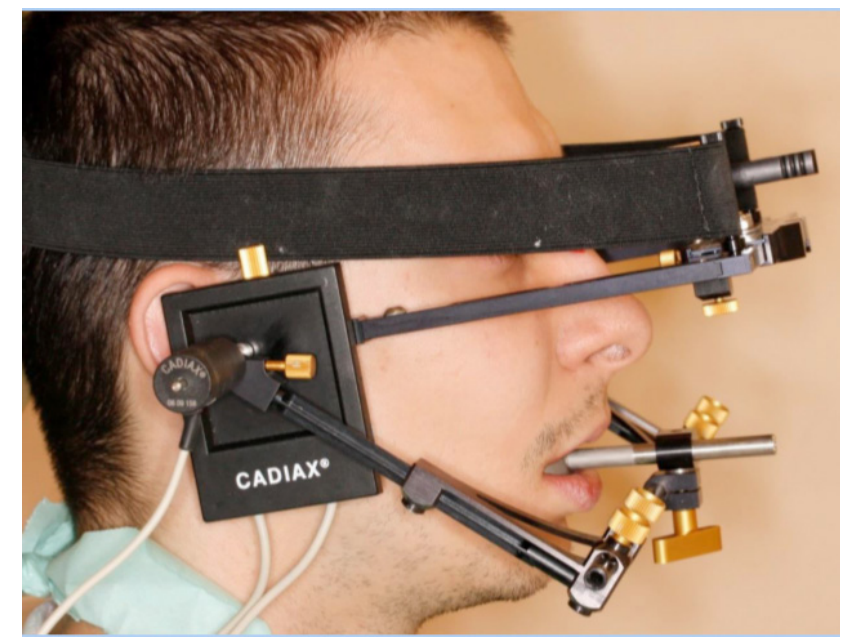

Figure 1. Axiograph application technique.

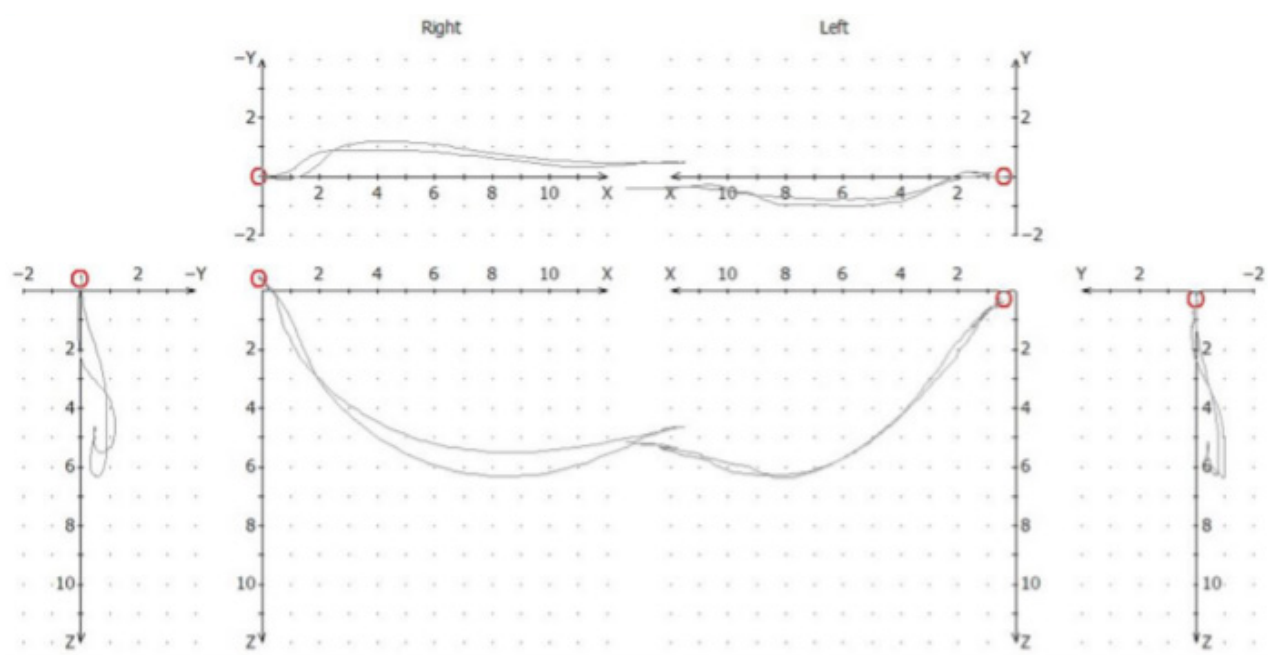

Figure 2. The graphs in Disc Displacement with reduction (DDwR) on the right side.

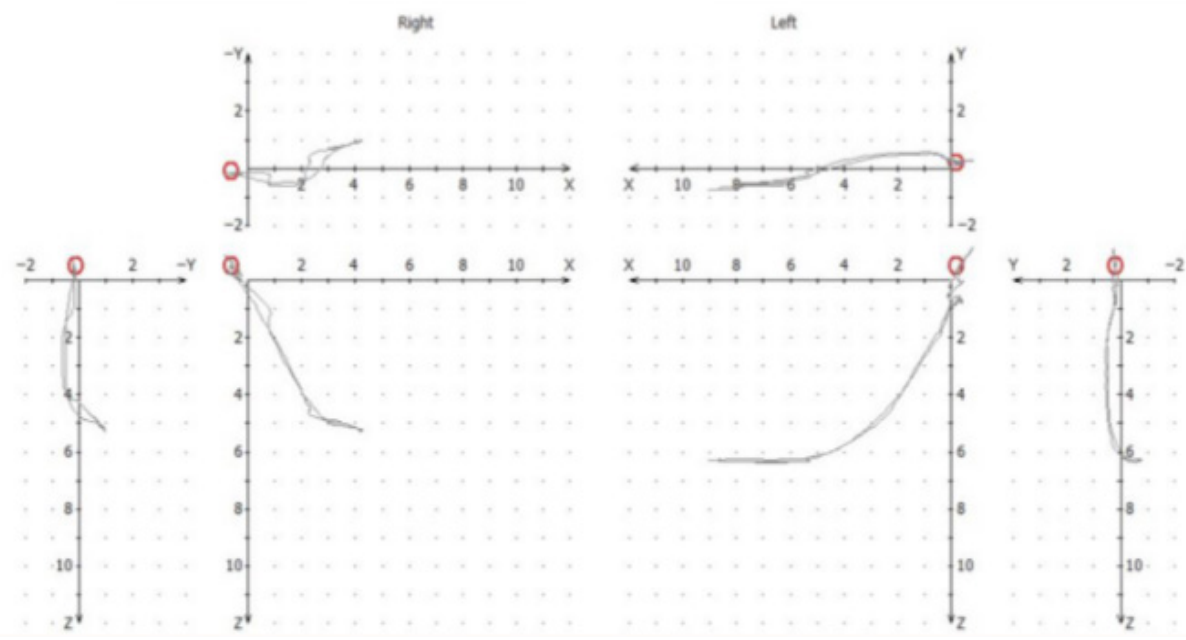

Figure 3. The graphs in Disc Displacement without reduction (DDwoR) on the right side. 


\section{Results}

Out of the 50 subjects, $31(62 \%)$ were females and $19(38 \%)$ males, aged between 21 and 62 years. The clinical diagnosis revealed that $27(54 \%)$ subjects were positive for a type of disk displacement and negative for $23(46 \%)$ subjects, while the diagnosis obtained through axiography was positive for $33(66 \%)$ subjects and negative for 17 $(36 \%)$ subjects (Figure 4).

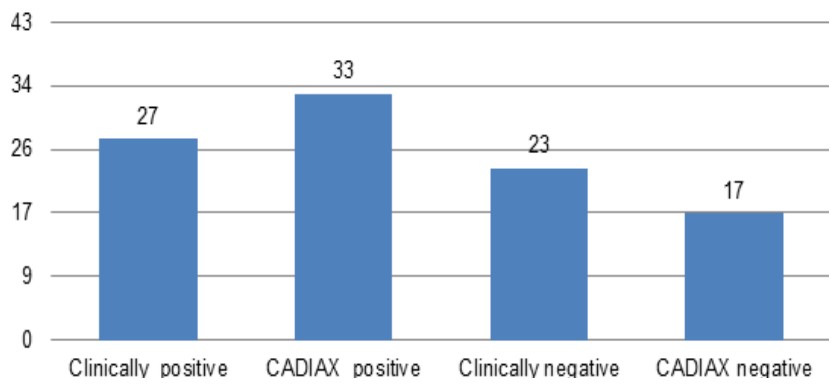

Figure 4. Distribution of subjects based on clinical and paraclinical diagnosis.

Following the clinical examination, out of the 50 patients, 20 patients $(42 \%)$ were diagnosed with disc displacement with reduction $(\mathrm{DDwR})(10$ of which with bilateral DDwR and 10 with unilateral DDwR), 6 patients $(12 \%)$ were diagnosed with disc displacement without reduction (DDwoR) (4 of which with unilateral DDwoR and 2 with bilateral DDwoR); no subluxation was diagnosed, and the remaining 23 patients (46\%) were considered to be clinically healthy (Figure 5).

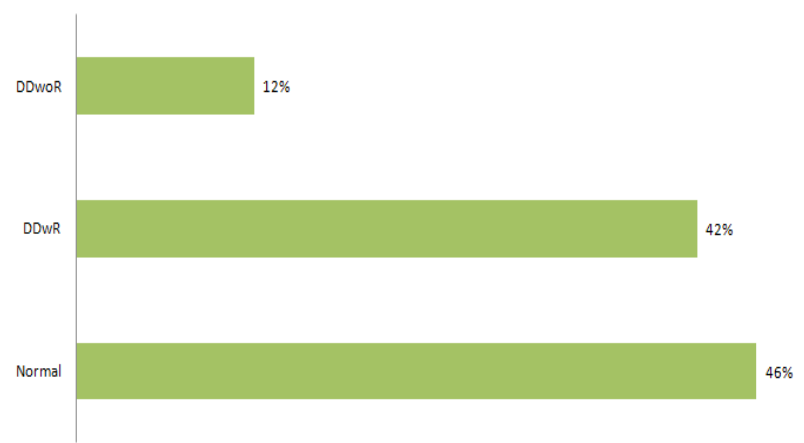

Figure 5. Distribution of subjects according to the clinical diagnosis.

After the paraclinical examination, using the CADIAX 2 axiograph, out of the 50 patients, $25(50 \%)$ were diagnosed with DDwR (14 of which with bilateral DDwR, and 11 with unilateral DDwR); 6 patients (12\%) were diagnosed with DDwoR (2 of which with bilateral DDwoR and 4 with unilateral DDwoR), 2 patients (4\%) had subluxation and 17 subjects did not present any symptoms/ signs of temporomandibular dysfunction (Figure 6).

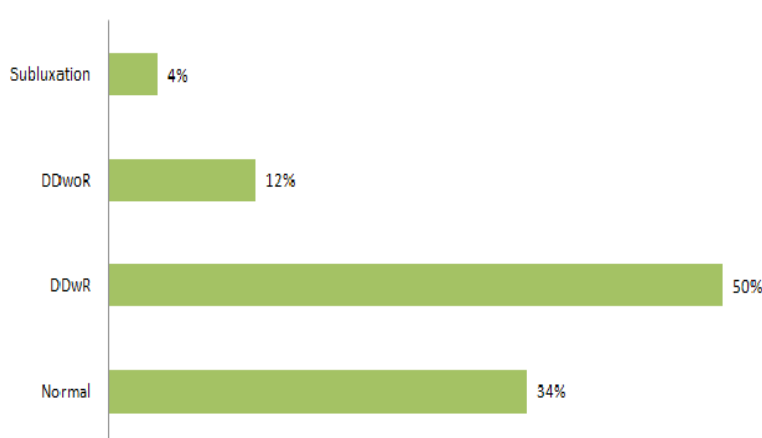

Figure 6. Distribution of subjects according to the CADIAX Compact 2 axiograph diagnosis.

As a percentage of positively diagnosed patients (DDwR, DDwoR, subluxation), the CADIAX compact 2 device diagnosed $66 \%$ of patients, compared to the clinical diagnosis where $54 \%$ were positively identified. Patients diagnosed negatively (i.e., without any kind of temporomandibular joint dysfunction) by the CADIAX device were $34 \%$, while clinical examination identified $46 \%$.

The contingency table shows a number of patients diagnosed as "truly positive" (TP) of 27 , "false positive" (FP) of 6, 0 "false negatives" (FN) and 17 "truly negatives" (TN) (Table I).

Table I. Contingency table of the subjects included in the study.

\begin{tabular}{|l|c|c|c|}
\multicolumn{4}{c}{ Clinical diagnosis } \\
\hline CADIAX diagnosis & Negative & Positive & Total \\
\hline Negative & 17.00 & 0.00 & 17.00 \\
Row\% & 1.00 & 0.00 & 1.00 \\
Col\% & 0.74 & 0.00 & 0.34 \\
Positive & 6.00 & 27.00 & 33.00 \\
Row\% & 0.18 & 0.82 & 1.00 \\
Col\% & 0.26 & 1.00 & 0.66 \\
TOTAL & 23.00 & 27.00 & 50.00 \\
Row\% & 0.46 & 0.54 & 1.00 \\
Col\% & 1.00 & 1.00 & 1.00
\end{tabular}

Pearson Chi-Square test (with McNemar's test) was applied, in order to check the validity of the paraclinical method using the Cadiax Compact 2. The statistical analysis revealed $\mathrm{p}=0.0412$.

\section{Discussion}

This retrospective study included 50 subjects with clinical symptoms of temporomandibular disorders, such as TMJ pain, clicking and/or crepitus, limited opening of the mouth. After a complete clinical examination for each patient (performed by a prosthodontic specialist with 20 years or experience) a clinical diagnosis was established. Afterwards, all subjects underwent computerized axiography using the CADIAX compact 
2 device, performed also by a prosthodontic specialist, with 8 years of experience in using the axiograph device. The subjects were clinically healthy individuals, without extended edentulous spaces, but displaying clinical signs of temporomandibular disorders. The inclusion and exclusion criteria were defined in a manner meant to exclude the influence of any systemic disorders on the function of the stomatognathic system. Also, the examiner performing the clinical investigation was highly trained in establishing a clinical diagnosis of TMD; at the same time, the examiner performing the axiography was also highly experienced in working with the equipment.

The CADIAX Compact 2, which was used for paraclinical examination, is a device that consists of the following elements: a face bow, a mandibular bow, a bite fork, two stilettos, two recording sensors, a central unit and a computer that contains the required software (Gamma Dental Software for Windows). The movements recorded with CADIAX Compact 2 were: the maximum protrusion, maximum lateral (right and left) movement, and maximum opening; each movement begin and end in the same reference position (centric relation) set as a starting point. The graph obtained may be instantly seen on the computer screen, and if the quality of the recording is questionable, the process may be repeated [3].

The presumptive diagnosis obtained after the muscular-articular clinical examination can be interpreted on the graphs obtained, according to the 3 spatial dimensions: the $\mathrm{X}$ and $\mathrm{Z}$ axes define the sagittal inclination of the condyle in the sagittal plane, and the angle is calculated between the condylar path and the Frankfurt plane. In the horizontal plane (the $\mathrm{X}$ and $\mathrm{Y}$ axes), the Bennet angle is calculated between the condylar path and the projection of the mid-sagittal plane $[13,14]$.

The present study has a sensitivity of $100 \%$, which means that the CADIAX device detected all clinically positive patients. On the other hand, the specificity of the study was $74 \%$, representing the percentage of patients negatively diagnosed by CADIAX device out of the total number of patients clinically diagnosed as negative. The $88 \%$ accuracy shows the proportion in which the CADIAX device provides a diagnosis equal to the clinical one.

The positive probability ratio $(\mathrm{PPR}=3.83$ ) refers to how many times a positive diagnosis established by the CADIAX device was more likely to occur in a patient diagnosed as clinically positive than in a clinically negative diagnosis. The negative probability ratio $(\mathrm{NPR}=0)$ depicts how often a negative diagnosis established by the CADIAX device was more likely to be found in a patient diagnosed as clinically positive than in a clinically negative diagnosis.

The extrinsic indicator PPV $=0.82$ or $82 \%$ (Positive Predictive Value) refers to the probability that a patient positively diagnosed with the CADIAX device does in fact suffer of temporomandibular dysfunction, or, in other words, it indicates the proportion of clinically positive patients in the number of patients diagnosed positively by
CADIAX device. The NPV indicator $=1$ or $100 \%$ (negative predictive value) indicates the probability that a patient negatively diagnosed with the CADIAX device does not show any clinical sign of temporomandibular dysfunction, i.e., it indicates the proportion of clinically negative diagnosed patients in the number of patients negatively diagnosed by the CADIAX device (Table II).

Table II. Extrinsic and intrinsic indicators (PPR - Positive probability ratio; NPR - Negative probability ratio; PPV - Positive predictive value; NPV - Negative predictive value).

\begin{tabular}{|l|c|c|}
\hline Sensitivity & $\mathbf{1 . 0 0}$ & $\mathbf{1 0 0} \%$ \\
\hline Specificity & 0.74 & $74 \%$ \\
Accuracy & 0.88 & $88 \%$ \\
PPR & 3.83 & \\
NPR & 0.00 & \\
PPV & 0.82 & $82 \%$ \\
NPV & 1.00 & $100 \%$
\end{tabular}

Computerised axiography was also used in other studies (Botos et al., Ikeda et al., Schierz et al., Ahangari et al.) which validate the accuracy of the device in the reproducibility of mandibular movements in the 3 planes. Nevertheless there are studies that consider further research to be necessary regarding the evaluation of the possibility of diagnosing TMJ dysfunction using a computerized axiograph $[2,4,8,9,10,13,14]$.

The present study reveals that clinical examination is not always an effective method of diagnosing temporomandibular dysfunction, even when the examiner has extensive experience in the field. In this regard, there are precise methods for investigating and diagnosing TMJ dysfunctions, such as cone-beam computer tomography (CBCT) or magnetic resonance imaging (MRI). Although these methods are more accurate than computerized axiography $[15,16]$, they are expensive and the patient does not always have access to them (most often due to the region in which they live).

The results of the study reveal the validity of this complementary examination method using the axiograph in patients suspected of temporomandibular dysfunction $(\mathrm{p}=0.0412, \mathrm{p}<0.05)$.

There is also another device, CADIAX Diagnostic axiograph, which, compared to the CADIAX Compact 2, records both the translational movement of the condyle, as well as the rotational one; the difference between the two systems is that the "diagnostic" system is equipped with two sensors, and that for CADIAX Compact version the Frankfurt plane is set as an arbitrary plane. Also, the "diagnostic" system uses the orbital plane, as a kinematic plane. The advantages of the "diagnostic" system seems to be more accurate regarding the differential diagnosis, and it is considered to be even a reliable method for determining the type of temporomandibular disorder $[1,3]$. 


\section{Conclusions}

The diagnosis obtained with the CADIAX Compact 2 device, with a high sensitivity (100\%), is very useful for screening temporomandibular joint disorders. However, the diagnosis established with the CADIAX Compact 2 has low specificity (74\%), thus lowering its usefulness in making an accurate diagnosis of the targeted pathology.

Due to the small sample of subjects (50) and the validation of diagnostic techniques for the device, it can be concluded that more detailed research on a larger group of patients is needed.

Also, in order to obtain an accurate recording of mandibular movements with the CADIAX device, a certain degree of knowledge regarding this system and of the sequence of recording steps is required.

Of all the methods for investigating temporomandibular joint dysfunction, axiography is not invasive, does not irradiate the patient, it is relatively easy to use and it provides functional information about the TMJ.

\section{References}

1. Nagata K, Hori S, Mizuhashi R, Yokoe T, Atsumi Y, Nagai W, et al. Efficacy of mandibular manipulation technique for temporomandibular disorders patients with mouth opening limitation: a randomized controlled trial for comparison with improved multimodal therapy. J Prosthodont Res. 2019;63:202-209.

2. Botos AM, Mesaros AS, Zimbran AI. The contribution of computerized axiography to the functional evaluation of the temporomandibular joint: a case report. Clujul Med. 2016;89:438-442.

3. Pascu L, Constantiniuc M, Lascu LM, Buduru S, Zaharia $\mathrm{A}$, Almăşan $\mathrm{O}$, et al. Axiography between classic and modern, computerized recording of mandibular movements [Romanian]. Clujul Medical. 2010;83:426-430.

4. Ikeda M, Miyamoto JJ, Takada JI, Moriyama K. Association between 3-dimensional mandibular morphology and condylar movement in subjects with mandibular asymmetry. Am J Orthod Dentofacial Orthop. 2017;151:324-334.
5. Buduru S, Tălmăceanu $\mathrm{D}$, Baru $\mathrm{O}$, Ureche M. Analiza ocluziei dentare - clinic versus articulator [Analysis of dental occlusion - clinical versus articulator]. Ed. Napoca Star Cluj, 2018.

6. Chang CL, Wang DH, Yang MC, Hsu WE, Hsu ML. Functional disorders of the temporomandibular joints: Internal derangement of the temporomandibular joint. Kaohsiung J Med Sci. 2018;34:223-230.

7. Dawson PE. Functional occlusion: from TMJ to smile design. Mosby Elsevier; 2007: pp 33-44, 45-56.

8. Ahangari AH, Torabi K, Pour SR, Ghodsi S. Evaluation of the Cadiax Compact ${ }^{\circledR}$ II accuracy in recording preadjusted condylar inclinations on fully adjustable articulator. J Contemp Dent Pract. 2012;13:504-508.

9. Schierz O, Klinger N, Schön G, Reissmann DR. The reliability of computerized condylar path angle assessment. Int J Comput Dent. 2014;17:35-51.

10. Schierz O, Wagner P, Rauch A, Reissmann DR. Impact of mounting methods in computerized axiography on assessment of condylar inclination. Cranio. 2019;37:60-67.

11. Choi BT, Hwang DY, Lee GH, Moon DN, Lee KM. Computerized ultrasonic axiographic evaluation of condylar movement in patients with internal derangement of the temporomandibular joint. Angle Orthod. 2019;89:924-929.

12. Kondrat W MD, Sierpinska T MD, PhD, Radke J MBA. Can devices for the instrumental analysis of temporomandibular joints be used interchangeably? Cranio. 2019;1-7.

13. Giraudeau A, Jeany M, Ehrmann E, Déjou J, Ouni I, JeanDaniel Orthlieb JD. Disc displacement without reduction: a retrospective study of a clinical diagnostic sign. Cranio. 2017;35:86-93.

14. Lomas J, Gurgenci T, Jackson C, Campbell D. Temporomandibular dysfunction. Aust J Gen Pract. 2018;47:212-215.

15. Madhavan S, Dhanraj M, Jain AR. Methods of recording mandibular movements - A review. Drug Invention Today. 2018;10:1254-1259.

16. Naqash TA, Chaturvedi S, Yaqoob A, Saquib S, Addas MK, Alfarsi M. Evaluation of sagittal condylar guidance angles using computerized pantographic tracings, protrusive interocclusal records, and 3D-CBCT imaging techniques for oral rehabilitation. Niger J Clin Pract. 2020;23:550-554. 